\title{
Effective Use of a Ready-made Ocular Prosthesis for Contracted Anophthalmic Socket Reconstruction Surgery
}

Hiroko Taneda* and Shigemi Sakai

Department of Plastic Surgery, International University of Health and Welfare Mita Hospital, Tokyo, Japan

\begin{abstract}
The use of an ocular prosthesis often helps to minimize the psychological problems associated with eye loss. Unfortunately, the anophthalmic socket sometimes contracts in patient's long life time. To solve this problem, an anophthalmic socket reconstruction is indicated and the reconstruction requires creating a cavity of the appropriate size and shape for retaining an ocular prosthesis. In a reconstruction surgery, to expand the contracted anophthalmic socket, an ocular conformer is commonly employed with a free skin graft. To create an anophthalmic socket cavity appropriate for an ocular prosthesis, we used a ready-made ocular prosthesis as the ocular conformer with a free skin graft. We believe that this method is optimal for creating the required hemispherical shell shape cavity in the anophthalmic socket in order to wear an ocular prosthesis successfully.
\end{abstract}

Keywords: Ocular prosthesis; Anophthalmic socket reconstruction; Socket contracture; Skin graft

\section{Introduction}

Successful reconstruction of the anophthalmic socket for retaining an artificial eye requires the creation of a cavity of sufficient size and shape [1]. Unfortunately, the anophthalmic socket sometimes contracts and the eye socket cavity shrinks. To rectify this problem, eye socket reconstruction surgery is often required. Several studies have reported the successful use of a free skin graft in anophthalmic socket reconstruction [2]. With a free skin graft, an ocular conformer is normally used to press the graft firmly onto the cavity surface and to maintain its position until healing has taken place [1]. This article describes an experience of a ready-made ocular prosthesis use as an ocular conformer. This method allows for the creation of a hemispherical shell shape of the anophthalmic socket cavity appropriate for maintaining an ocular prosthesis.

\section{Case Report}

A 68-year-old male patient was referred to the Department of Plastic Surgery, International University of Health and Welfare Mita

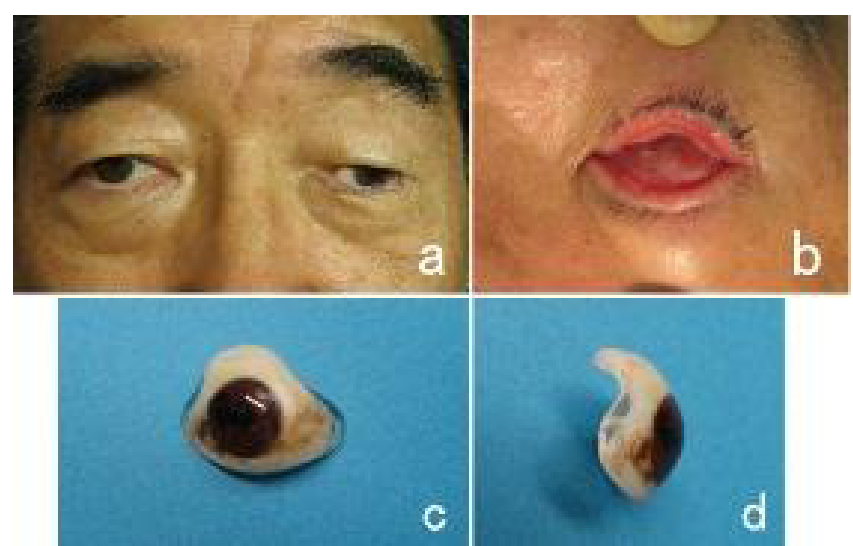

Figure 1: A 68-year-old male patient. Due to the inflammation caused by the measles at the age of three, the patient lost sight in his left eye, and the eye finally had to be enucleated.

a. Preoperative photograph

b. His left eye socket had shrunk, and the patient experienced with his ocular prosthesis slipping down due to the shallow lower fornix.

$c$ and d. Photographs of the prosthesis that was distorted in shape and became smaller.
Hospital, Tokyo, for eye socket reconstruction surgery. He had suffered from measles at the age of three. Due to the inflammation caused by the measles, the patient lost sight in his left eye, and the eye finally had to be enucleated (Figure 1a). His left eye socket had shrunk gradually, and he experienced the recent difficulty of maintaining ocular prosthesis. Therefore, he had tried to reduce in the size of his ocular prosthesis. In spite of the reduction of ocular prosthesis size, the difficulty was unimproved and the prosthesis was distorted in shape and became smaller and smaller (Figure 1c and 1d). In addition, the ocular prosthesis was easily slipping down due to the shallowness of lower fornix (Figure 1b).

\section{Surgical Procedure}

First, a new cavity was created by dissecting the skin at the bottom of the eye socket (Figure 2a). Skin for the free graft was harvested from the groin area. This skin was grafted into the cavity to form the new eye socket (Figure 2b). In order to create a stable and deep lower fornix, the lower edge of the skin was sutured to the inferior orbital bone rim using anchor sutures (Figure 2c). These anchor sutures served to maintain sufficient depth of the lower fornix in the new eye socket so that the patient would be able to retain the eye prosthesis (Figure 2d). The anchor sutures were secured with bolster sutures with a small degree of slack to accommodate the post-surgical swelling. One week later, the sutures were removed.

The temporary ocular prosthesis remained in place until healing was complete, which is usually six months to ten months following surgery. After this time, the patient was fitted with a new, custom-made ocular prosthesis.

*Corresponding author: Hiroko Taneda, Department of Plastic Surgery, International University of Health and Welfare Mita Hospital, 1-4-3 Mita, Minatoku, Tokyo 108-8329, Japan, Tel: +81-3-3451-8121; Fax: +81-3-3454-0067; E-mail: hiro0617@oita-u.ac.jp

Received February 25, 2013; Accepted March 11, 2013; Published March 14 2013

Citation: Taneda H, Sakai S (2013) Effective Use of a Ready-made Ocular Prosthesis for Contracted Anophthalmic Socket Reconstruction Surgery. Anaplastology 2: 107. doi: 10.4172/2161-1173.1000107

Copyright: ( 2013 Taneda $\mathrm{H}$, et al. This is an open-access article distributed unde the terms of the Creative Commons Attribution License, which permits unrestricted use, distribution, and reproduction in any medium, provided the original author and source are credited. 
Citation: Taneda H, Sakai S (2013) Effective Use of a Ready-made Ocular Prosthesis for Contracted Anophthalmic Socket Reconstruction Surgery. Anaplastology 2: 107. doi: 10.4172/2161-1173.1000107
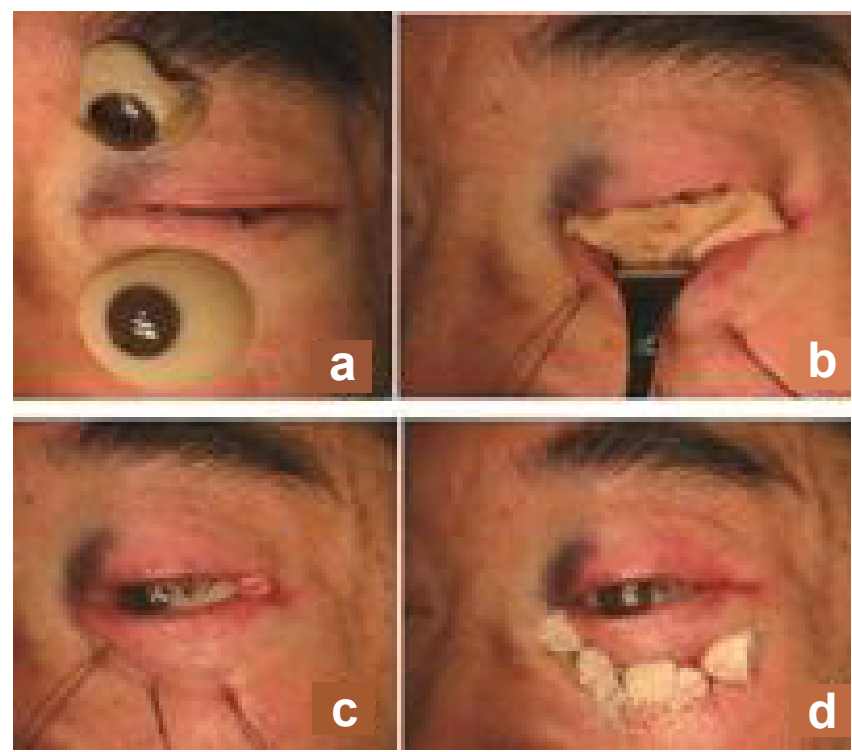

Figure 2: Intraoperative photographs.

a. A new cavity was created by dissecting the skin at the bottom of the eye socket the upper ocular prosthesis was his original eye prosthesis. The lower ocular prosthesis was ready-made eye prosthesis as ocular conformer. b.

This skin was grafted into the cavity to form the new eye socket

c. In order to create a stable and deep lower fornix, the lower edge of the skin was sutured to the inferior orbital bone rim using anchor sutures.

d. These anchor sutures served to maintain sufficient depth of the lower fornix in the new eye socket so that the patient would be able to retain the eye prosthesis.
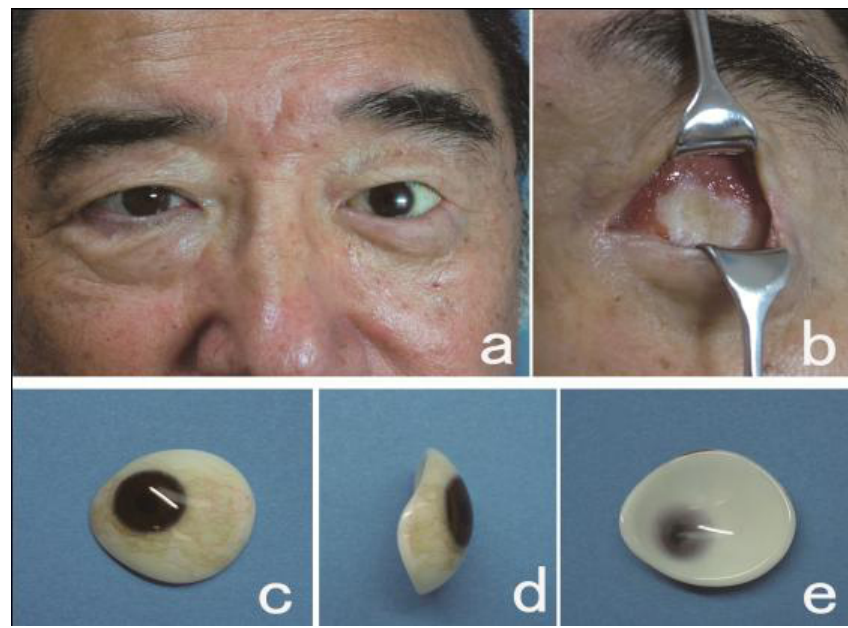

Figure 3: Sixty-six months following surgery.

$a$ and $b$. The patient still maintained proper cavity of sufficient size and shape for wearing his new prosthesis (postoperative view).

c-e. The new prosthesis was a thin and had a hemispherical shell shape.

One year following surgery, the patient was fitted with a new, custom-made ocular prosthesis. Sixty-six months following surgery, the patient still maintained proper cavity of sufficient size and shape for wearing his prosthesis (Figures $3 \mathrm{a}$ and $3 \mathrm{~b}$ ). The new prosthesis was thin and had a hemispherical shell shape (Figures 3c-3e). The patient reported that ocular hygiene was much easier to maintain and that his mental state had improved.

\section{Discussion}

Successful eye socket reconstruction requires creating a cavity of the appropriate size and shape for retaining an ocular prosthesis [1]. The optimum ocular prosthesis is thin and hemispherical shell shaped. A thin and lightweight ocular prosthesis does not increase the strain on the eye lid tissue, especially not on the lower eyelid. Moreover, a smooth shaped eye socket cavity is easy to keep clean.

Using a ready-made ocular prosthesis as an ocular conformer for surgery may represent the optimal approach for creating an appropriate eye socket cavity capable of retaining an ocular prosthesis. This would require, however, that various sizes and shapes of ready-made prostheses be on hand during the surgery.

\section{Conclusion}

For contracted eye socket reconstruction, the use of a ready-made ocular prosthesis as an ocular conformer is optimal for helping the surgeon create a hemispherical shell shape cavity in the anophthalmic socket that would be capable of retaining an ocular prosthesis successfully.

\section{References}

1. Converse JM (1977) Reconstructive plastic surgery. (2ndedition), The orbit. W. B. Saunders, Philadelphia.

2. Antia NH, Arora S (1984) "Malignant" contracture of the eye socket. Plast Reconstr Surg 74: 292-294. 